Revue d'histoire de l'Amérique française

REVUE D.HISTOIRE DE L'AMÉRIQUE FRANÇAISE

\title{
Projet d'invasion du Canada au début de 1778
}

Lafayette, commandant en chef du corps expéditionnaire américain

\section{Marcel Trudel}

Volume 2, numéro 2, septembre 1948

URI : https://id.erudit.org/iderudit/801448ar

DOI : https://doi.org/10.7202/801448ar

Aller au sommaire du numéro

\section{Éditeur(s)}

Institut d'histoire de l'Amérique française

\section{ISSN}

0035-2357 (imprimé)

1492-1383 (numérique)

Découvrir la revue

\section{Citer cet article}

Trudel, M. (1948). Projet d'invasion du Canada au début de 1778 : Lafayette, commandant en chef du corps expéditionnaire américain. Revue d'histoire de l'Amérique française, 2(2), 163-184. https://doi.org/10.7202/801448ar d'utilisation que vous pouvez consulter en ligne. 


\title{
PROJET D'INVASION DU CANADA AU DEBUT DE 1778:
}

\author{
LAFAYETTE, COMMANDANT EN CHEF \\ DU CORPS EXPEDITIONNAIRE \\ AMERICAIN*
}

Les volontaires français commencèrent bien avant l'alliance de 1778 à franchir l'Atlantique pour se mettre au service des treize colonies révoltées contre l'Angleterre. Dès le printemps de 1775, avant même la bataille de Bunker Hill, il s'en trouvait dans l'armée américaine. Ils continuèrent par la suite à se présenter de plus en plus nombreux. Silas Deane, délégué en France par le Congrès au printemps de 1776 et Benjamin Franklin qui était allé le rejoindre quelques mois plus tard ${ }^{1}$, furent littéralement assaillis par une nuée de candidats qui brûlaient de se battre pour la défense de la liberté ou... pour conquérir des grades. Nobles, pour la plupart, très souvent sans aucune expérience militaire et presque toujours sans aucune connaissance de l'anglais, ils exigeaient une solde mirobolante et les fonctions militaires les plus importantes, persuadés que l'armée américaine manquait absolument d'officiers pour la diriger. Les Commissaires du Congrès, pour ménager les susceptibilités d'un pays dont ils venaient réclamer l'assistance, et les noms illustres sur lesquels s'appuyaient les candidats, se voyaient contraints d'accepter les conditions qu'on leur proposait. Or ces volontaires, quand ils arrivaient en Amérique, éprouvaient les déceptions les plus amères: le Congrès refusait de reconnaitre des contrats d'enrôlement qui étaient une injustice à l'égard des officiers réguliers de l'armée américaine ou qui ne pouvaient avoir aucune valeur

*Extrait d'un ouvrage en préparation: Louis XVI, le Congrès américain et le sort du Canada (1774-1789).

1. Il débarqua à Nantes dans les premiers jours de décembre 1776. 
pratique parce que les postulants ignoraient la langue anglaise. On n'avait demandé à Deane et à Franklin que des officiers du génie et d'artillerie; on se trouvait maintenant débordé par des officiers de toute catégorie qui se montraient insupportables dans leurs réclamations et vantards comme Gascons ${ }^{2}$. Le Congrès en accepta un certain nombre, il renvoya les autres en leur remboursant les frais de voyage pour l'aller et pour le retour ${ }^{3}$.

Parmi ces volontaires, il s'en trouve trois qui, passés à peu près en même temps au service du Congrès, sont restés célèbres à des degrés inégaux et pour des raisons différentes: du Coudray, Conway et Lafayette. Philippe Tronson du Coudray, officier d'artillerie, signait par-devant Deane, le 7 décembre 1776, un contrat qui lui conférait le grade de major-général de l'armée américaine, le commandement de toute l'artillerie, le pouvoir de remplir les postes vacants et le privilège d'être indépendant de tout autre supérieur que Washington et le Congrès; sa solde et celle des officiers qui l'accompagnaient devaient compter à partir du mois d'aout précédent; le contrat assurait de plus une pension à vie égale à une demi-solde ${ }^{4}$. Embarqué sur l'Amphitrite que Beaumarchais avait eu tant de peine à faire partir en secret, il se déclare mécontent du logement, fait revenir le navire au port: le gouvernement français, pour apaiser les protestations de l'ambassadeur anglais contre la contrebande des armes, ordonne que toute la cargaison soit déchargée. Parvenu enfin aux Etats-Unis, du Coudray soulève l'indignation des Américains à cause des avantages surprenants qu'il s'est fait accorder par les Commissaires: plusieurs hauts officiers menacent de démissionner si le contrat n'est pas rescindé. Sa mort accidentelle, le 15 septembre 1777 , met fin à l'imbroglio.

Thomas Conway était un officier irlandais passé depuis longtemps au service de la France. Arrivé en Amérique, il avait réussi, à la

2. Letters of Members of the Continental Congress (édition Burnett), t. II, p. 394 et 439. Cette collection sera dans la suite désignée par le sigle LMCC. FrankLIN, Writings (éd. Smyth), t. VII, p. 61, 77, 81s.

3. Le renvoi de ces volontaires fut pour le Congrès l'un des problèmes financiers les plus compliqués et les plus coûteux de l'année 1777. Voir en particulier les tomes VIII et IX des Journals of the Continental Congress (collection présentée désormais sous le sigle JCC).

4. The revolutionary diplomatic correspondence of the United States (éd. Wharton), t. I, p. 82s. Publication citée ci-après RDCor. 
grande indignation des officiers qu'il dépassait ainsi sans suivre la filière traditionnelle, à se faire nommer brigadier-général en mai 1777; lorsqu'il sollicita du Congrès (où il avait beaucoup d'amis) le grade de major-général, Washington ne put cette fois s'empêcher de manifester ouvertement son opposition: ce qui eut pour effet de faire adopter à Conway le parti des adversaires de Washington. Aussi imprudent qu'ambitieux, il se permit des remarques désagréables sur la stratégie et sur la valeur du général en chef. Une de ses lettres tomba entre les mains de Washington qui laissa savoir, de la façon la plus sèche, qu'il était au courant de tout ${ }^{5}$. C'est là l'un des épisodes de ce qu'on a appelé la Conway cabal qui, sous l'instigation du général Horatio Gates, fut la tentative la plus sérieuse pour remplacer Washington. C'est à cette cabale qu'il faut rattacher le projet d'invasion du Canada en 1778.

Quant au marquis de Lafayette ${ }^{6}$, il n'avait pas agi avec le même égoïsme. Dans toute la collaboration officielle ou privée de la France, c'est lui, l'adolescent de dix-neuf ans, qui représente le véritable esprit chevaleresque, et c'est pourquoi les Américains lui ont voué une sorte de culte ${ }^{7}$. Membre de l'une des familles les plus influentes et les plus riches de France (il était neveu des Ségur et avait épousé une d'Ayen), tout lui promettait en France l'avenir le plus facile et le plus brillant, mais il préféra conquérir de haute lutte les honneurs et la gloire. Le même jour que du Coudray, il signe devant Deane son contrat d'enrôlement: certes, il a soin, pour s'assurer un avancement plus rapide en France le jour où celle-ci aura reconnu les Etats-Unis, de se faire garantir le grade de major-général; mais alors que du Coudray et les autres exigent une solde très élevée, il s'engage à " servir les dits Etats avec tout le zèle possible sans aucune pension ni traitement particulier " ${ }^{8}$; en mars 1777 , il frète à ses frais un navire qu'il a

5. Lettre a Conway, le 9 novembre 1777: Washington, Writings (éd. Fitzpatrick), t. X, p. 29.

6. Marie-Paul-Joseph-Roch-Yves-Gilbert de Motier, marquis de Lafayette (1757-1834).

7. La visite qu'il viendra faire aux Etats-Unis en 1824 tournera en une longue promenade de triomphe qui laissera dans l'ombre les élections présidentielles ellesmêmes. Louis XVI, Vergennes et Beaumarchais ne furent jamais vraiment populaires auprès des Américains.

8. Le texte du contrat de Lafayette, à la date du 7 décembre 1776, est reproduit dans les Collections of the Connecticut Historical Society, t. XXIII, p. 54s. 
acheté, il le charge de munitions et, après avoir déjoué la police du roi qui lui court après pour l'empêcher de partir, il file vers l'Amérique.

Lafayette ne manquait ni de feu ni d'enthousiasme; le comte de Ségur pourra dire du marquis et de ses amis: "Comme c'était pour la liberté que la guerre se faisait alors entre les Américains et les Anglais, cette même liberté s'offrait à nous avec tous les attraits de la gloire; et, tandis que des hommes plus mûrs et les partisans de la philosophie ne voyaient dans cette grande querelle qu'une favorable occasion pour faire adopter leurs principes... nous, plus jeunes, plus légers et plus ardents, nous ne nous enrôlions sous les enseignes de la philosophie que dans l'espoir de guerroyer, de nous distinguer, d'acquérir de l'honneur et des grades; enfin, c'était comme paladins que nous nous montrions philosophes. " ${ }^{9}$ Lafayette ne fut cependant pas l'écervelé que la tradition ou l'imagination romanesque se plaît à représenter, se lançant dans une aventure audacieuse à la suite d'un coup de tête et donnant libre cours à l'imprudence de ses vingt ans. Si l'on veut comprendre comment Washington, général en chef de l'armée américaine et âgé de quarante-cinq ans, dont le maintien froid glaçait les intimes et qui s'épanche si rarement dans sa volumineuse correspondance, ait pu ressentir tant d'amitié et de confiance à l'égard d'un jeune homme qui n'avait pas encore atteint sa majorité, il faut bien retenir cette phrase du comte de Ségur: "Lafayette eut de tout temps, surtout quand il était jeune, un maintien froid, grave et qui annonçait même très faussement une apparence d'embarras et de timidité... mais cette enveloppe si froide aux regards, cachait l'esprit le plus actif, le caractère le plus ferme et l'âme la plus brûlante. ") ${ }^{10}$ Aussi lorsque le " young boy " (expression favorite de lord Cornwallis) se présenta devant le Congrès pour faire reconnaître son titre de majorgénéral et réclamer le commandement d'une division de l'armée, c'est Washington lui-même, ce général qu'on accusait toujours de prudence excessive, qui intervint en faveur de Lafayette et proposa au Congrès de confier des troupes au jeune officier ${ }^{11}$.

9. SEgur, Mémoires, souvenirs et anecdotes, t. I, p. 87. Ségur, ami intime de Lafayette et du même âge que lui, se trouvait être aussi l'oncle du jeune marquis; il projeta en vain de venir en Amérique en même temps que Lafayette.

10. Stgur, op. cit., p. 72.

11. Washington au président du Congrès, le $1^{\mathrm{er}}$ novembre 1777: Writings, t. IX, p. 480 . 
Dans le temps où Lafayette faisait ses débuts en Amérique, la nouvelle république se trouvait placée dans la position la plus dangereuse: Burgoyne s'avançait par le nord contre les positions essentielles de la rivière Hudson, pendant que Howe, de New-York, devait aller le rejoindre à Albany avec une armée formidable, afin d'isoler la Nouvelle-Angleterre et de s'assurer par la vallée de l'Hudson le contrôle des Grands Lacs et tout le commerce de l'Ouest. Mais l'avance victorieuse de Burgoyne s'était changée en un désastre et, le 17 octobre 1777, Burgoyne mettait bas les armes et capitulait avec toute son armée. Jamais pendant toute la guerre de Sept Ans, on n'avait vu une armée entière se faire capturer de la sorte, et cela par une armée de colons organisés en milice et dirigés par des chefs à peu près inconnus. La victoire de Saratoga modifiait profondément la position des Américains: Burgoyne passait pour être le général le plus habile d'Angleterre et l'on savait en même temps que l'ancienne mère-patrie ne pourrait jamais lancer contre la république américaine plus de troupes qu'elle l'avait fait jusqu'alors; de plus, la France qui collaborait en secret depuis 1775, se trouvait rassurée sur le destin des Etats-Unis et acceptait d'entrer officiellement en pourparlers avec les Commissaires du Congrès ${ }^{12}$. La république pouvait maintenant passer de la défensive à l'offensive: la victoire de Saratoga permettait de songer de nouveau à la conquête du Canada.

Depuis la débâcle de juin 1776, on avait toujours songé à reprendre le Canada. Aussi longtemps qu'il serait britannique, pensait-on, l'existence des Etats-Unis se trouvait menacée. Le Canada devait être conquis ou confédéré. Tous les plans de confédération soumis au Congrès depuis 1776 réservaient un article spécial à la colonie canadienne. Le 12 juillet 1776, cet article était ainsi conçu: "Le Canada accédant à cette confédération et se joignant entièrement aux mesures des Colonies-Unies sera admis à, et jouira de, tous les avantages de cette union. Mais aucune autre colonie ne sera admise dans la dite union, à moins que son admission ne soit approuvée par les délégués de

12. La nouvelle de la victoire américaine parvint en France au début de décembre 1777: Vergennes, Secrétaire d'État de Louis XVI, qui jusque alors avait tenu à l'écart Deane et Franklin, leur fit présenter les félicitations du roi. Les Commissaires en profitèrent pour proposer un traité d'alliance en insinuant habilement qưe l'Angleterre songeait à faire des propositions de paix. 
neuf colonies. " ${ }^{13}$ Ce traitement de faveur fut encore adouci après la victoire de Saratoga, lorsque, concernant l'adoption des mesures du Congrès par le Canada, on supprima le mot "entièrement " ${ }^{14}$. Après avoir décidé d'accorder au Canada ce traitement spécial qu'on refusait a d'autres colonies voisines, le Congrès invita un comité à publier en français ces articles de confédération, à " présenter une adresse aux habitants du Canada, les invitant à accéder à l'union de ces Etats " ainsi qu'un plan " pour faciliter la distribution des dits articles et adresse et pour concilier les amitiés des Canadiens envers ces EtatsUnis $)^{15}$. Le 2 décembre, ce comité déposait son rapport ${ }^{16}$ : il fallait, proposait-on, construire sur le lac Champlain une forteresse qui contienne une garnison de 700 hommes, bien pourvus de vivres et de munitions; on enrôlerait sous les drapeaux américains les prisonniers canadiens qui y consentiraient ${ }^{17}$ et généralement tout Canadien ou Français afin de constituer une légion française; pour encourager le recrutement de ces volontaires, il fallait assurer des promotions dans l'armée à tout Canadien qui amènerait des hommes ${ }^{18}$ : cette légion aurait pour chapelain, désigné par le commandant, " tout prêtre canadien, ministre ou diacre de bonne réputation, qui l'aidera à compléter le dit bataillon et à promouvoir l'accession du Canada à l'union de ces Etats ") ; enfin, on proposait de donner au commandant de cette légion l'autorisation de préparer les habitants du Canada à opérer une révolution quand les Etats-Unis en jugeraient l'heure arrivée. Le comité ne parla pas d'adresser une proclamation aux

13. Secret journals of the acts and proceedings of Congress, t. I, p. 287. Ce titre reparaitra sous le sigle SJCC.

14. L'article du Canada qui, le 12 juillet 1776, se trouvait l'article $X X$, devient l'article XVI, le 20 aodt suivant (SJCC I 298), puis l'article XI, le 15 novembre 1777 (SJCC I 345); il reste l'article XI dans le plan de confédération approuvé officiellement le $1^{\text {er }}$ mars 1781 (SJCC I 445).

15. Le 29 novembre 1777. Le comité était composé de William Duer, Francis Lightfoot Lee et James Lovell (SJCC I 452).

16. Pour le texte de ce long rapport, voir JCC IX 985-988.

17. Il s'agit des Canadiens qu'on avait faits prisonniers lors de l'invasion de 1775-1776 et de ceux qui se troưvaient dans l'armée de Burgoyne (rappelons que sur les 6,840 soldats de Burgoyne, il n'y avait que 148 Canadiens).

18. Avec trente volontaires, le recruteur devenait sous-lieutenant; avec 15, il devenait enseigne; avec 5, sergent; avec 3, caporal (JCC IX 986-987). 
Canadiens comme le Congrès en avait exprimé le désir, le 29 novembre précédent.

Si dans la suite le Congrès semble renoncer aux mesures qu'avait proposées le rapport Duer-Lee-Lovell ${ }^{19}$, il n'en prend pas moins des décisions bien agressives à l'égard du Canada. Dès le 3 décembre, il veut lancer un parti d'incursion contre Saint-Jean: Stark est invité a lever un certain nombre de volontaires et, pour les approvisionner, à obtenir de Gates, commandant en chef du département du nord, les magasins militaires suffisants et cinq mille dollars; l'objectif de l'incursion était de détruire les barges britanniques à Saint-Jean ou ailleurs sur la lac Champlain et l'on offrait vingt mille dollars si l'expédition était fructueuse; ou, si les volontaires préféraient ne pas prendre le risque de perdre toute récompense en cas d'insuccès, on offrait de doubler la solde pour la durée de l'incursion. James Duane, délégué du New-York au Congrès général, fut chargé d'aller communiquer à Stark le projet contre Saint-Jean ${ }^{20}$. Le 16 décembre, dans la correspondance de Duane, il n'est encore question que d'une simple incursion ${ }^{21}$, mais ce même projet allait bientôt se transformer en un plan beaucoup plus vaste et plus audacieux: l'incursion allait devenir en janvier 1778 une invasion proprement dite, par suite des intrigues d'un général ambitieux.

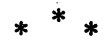

Horatio Gates, ex-officier britannique, avait toujours été favorisé par la bonne fortune. Nommé adjudant-général de l'armée américaine en juin 1775 , on l'avait désigné en juin 1776 pour commander l'armée du Canada avec des pouvoirs de " dictateur romain " 22 dans l'espoir qu'il réussirait à arrêter la débâcle américaine, mais la déroute

19. L'étude de ce rapport fut remise au 7 , mais on n'en parla que le 19 pour la remettre au 22; le 22, on la remet à plus tard, sans autre précision. Dans la suite, les Journals n'en parlent plus' (JCC IX 988-1049).

20. SJCC I 54-55.

21. Duane à Horatio Gates et à Philip Schuyler, le même jour (LMCC II 590).

22. C'est le 18 juin que John Adams écrivit à Gates qu'il était nommé pour le Canada avec des pouvoirs dictatoriaux pour une durée de six mois (LMCC I 497). C'était le moment où l'armée américaine repassait déjà les frontières. 
de l'armée d'invasion s'était accomplie avec une telle rapidité que Gates n'avait pas même eu le temps d'aller prendre son poste: il sortait donc indemne de toute cette affaire, alors que Schuyler, qu'il détestait, devait porter la responsabilité de l'échec, à titre de général en chef de l'armée d'invasion ${ }^{23}$. Survient l'expédition de Burgoyne: Schuyler, toujours à la tête du département du nord, doit reculer devant un ennemi qui lui est bien supérieur en nombre; il abandonne Ticonderoga que l'on considérait (à tort d'ailleurs) comme la clé de toute la défense des frontières du nord; il laisse tomber d'autres forts et l'ennemi s'avance toujours victorieux: Schuyler se voit alors enlever son commandement qu'on donne à Gates. Or les retraites successives opérées par Schuyler avaient eu pour résultat de faire entrer Burgoyne dans un vaste piège ${ }^{24}$; Gates n'avait plus qu'à terminer une besogne déjà fort avancée; il la termina, mais encore là, sa part reste fort mince: la fameuse victoire de Bennington revient toute à Stark ${ }^{25}$; c'est Benedict Arnold, laissé délibérément de côté par Gates, qui fait tourner en déroute l'expédition de Saint-Léger ${ }^{26}$; quant aux deux batailles de Freeman's Farm qui ont déterminé la déconfiture de Burgoyne à Saratoga, c'est encore à Benedict Arnold que revient le mérite d'en avoir fait des victoires, et cela malgré l'opposition de Gates lui-même ${ }^{27}$. Gates, cependant, dans les bulletins qu'il publiait, ne parlait jamais que de lui-même. Aux yeux du public et du Congrès,

23. Schuyler, malade à Ticonderoga, ne put jamais venir au Canada pendant l'invasion; il s'y fit remplacer par Montgomery d'abord et ensuite par David Wooster; comme ce dernier fut exonéré de tout blâme lors de l'enquête tenue par le Congrès sur l'échec du Canada, Schuyler comprit que c'était lui qu'on voulait condamner: il s'ensuivit de vives polémiques.

24. Burgoyne s'avançait à travers une région marécageuse et désolée; plus il allait de l'avant, plus son armée s'affaiblissait, épuisée par une marche extrêmement pénible (on ne pouvait faire plus d'un mille par joưr), menacée sur la gauche par la milice de la Nouvelle-Angleterre, ayant en face une armée entraînée à la petite guerre; et Burgoyne était toujours sans nouvelles de Howe qui devait venir prendre les Américains à revers. La défaite de Burgoyne fut prévue dès juillet.

25. Bataille du 16 aoât au cours de laquelle Stark extermina un corps d'armée allemand de 1,000 hommes en ne perdant qu'une quinzaine des siens.

26. Saint-Léger devait aller opérer sa jonction a Albany avec Howe et Burgoyne en descendant la Mohawk. Il dut retraiter précipitamment le 22 aołat.

27. Batailles du 19 septembre et du 7 octobre. Tenu à l'écart de tout commandement, Arnold se mit à la tête de troupes que Gates laissait inutilement dans l'inaction et, en intervenant à l'heure critique, obligea l'ennemi à retraiter dans l'une et l'autre bataille. 
la victoire de Saratoga, qui changeait tout le cours de la guerre, devenait l'œuvre de Gates; elle faisait de lui le général le plus en vue, plus en vue même que Washington, l'ami des Schuyler et des Arnold, puisque, au moment où Gates ne connaissait que des triomphes, Washington, dans le sud, ne subissait que des défaites et des reculs dans sa campagne défensive contre Howe. Comme s'ils oubliaient ou ignoraient que cette même campagne, en empêchant Howe d'aller faire sa jonction à Albany, assurait la défaite de Burgoyne, plusieurs officiers de l'état-major (et nous avons vu que Conway était de ceuxlà) ne manquèrent pas d'élever la voix contre la faiblesse de Washington et Gates devint ainsi le rival du général en chef. Elevé par le Congrès à la présidence du Bureau de la Guerre ${ }^{28}$, il obtint (toujours sous la dépendance de Washington, il est vrai) le contrôle de toutes les forces militaires. C'est alors qu'il conçut le projet d'ajouter à ses lauriers de Saratoga ceux du Canada et de conquérir toute une partie de l'Amérique pendant que Washington se trouvait tristement immobilisé à Valley Forge.

Sans avoir au préalable consulté le général en chef, Gates obtint du Congrès l'autorisation de transformer le plan d'incursion de décembre en un plan d'invasion proprement dite ${ }^{29}$ et, le 23 janvier 1778 , le Bureau de la Guerre procéda à l'élection des officiers; on résolut de donner la direction des troupes à des officiers français, officiellement pour en imposer davantage à la population française du Canada, mais en réalité pour faire passer dans le parti de Gates tous ces nobles venus de France, y compris le plus influent d'entre eux, le marquis de Lafayette, qu'on savait protégé par Washington: si l'invasion réussissait, ces officiers français ne manqueraient pas de manifester une grande reconnaissance à Gates pour l'occasion magnifique qu'il leur aurait donnée de se distinguer; si elle échouait, les amis de Washington, et Lafayette plus que les autres, se trouveraient compromis ${ }^{30}$. Le scrutin désigna au commandement de l'invasion le marquis de Lafayette; on lui donna comme second le major-général Conway ${ }^{31}$. Mais La-

28. Le 27 novembre 1777.

29. JCC X: le 22 janvier 1778.

30. Friederich KAPP, The life of John Kalb (New York, 1884), p. 149-150.

31. JCC X 87 . 
fayette, malgré son jeune âge, avait déjà la prudence des vieux officiers: le plan de Gates allait être déjoué.

Lafayette hésita d'abord à accepter le commandement de l'invasion, parce que ce commandement se trouvait confié à deux officiers venus de France et surtout parce que le second était Conway. Lafayette n'aimait pas Conway au moins depuis la cabale qui avait éclaté contre Washington l'automne précédent; il redoutait les ambitions de cet intrigant, ainsi que le démontre une lettre de Gouverneur Morris au président du Congrès: " Il vaut la peine que le Congrès considère si, au cas où il arrivait un accident au commandant en chef ${ }^{32}$, il serait prudent de laisser à une personne ${ }^{33}$ dont l'objet est de pousser sa fortune en France, l'opportunité d'inspirer de l'affection aux Canadiens envers le Grand Monarque qui aime peut-être le Canada autant que n'importe lequel de ses prédécesseurs ${ }^{34}$ "); et Morris ajoutait que cette considération venait du marquis lui-même ${ }^{35}$. Le problème était délicat: les Américains voulaient conquérir le Canada pour eux-mêmes évidemment; or les officiers français pouvaient très facilement être soupçonnés de vouloir le conquérir pour la France, sans compter que si l'invasion échouait, le prestige de la France se trouverait compromis. Lafayette exigea donc qu'on lui adjoignit comme second un major-général de l'armée américaine qui fút son successeur éventuel, MacDougall ou de Kalb ${ }^{36}$; il demanda de plus que la nomination des officiers fút faite par l'intermédiaire de Washington. Gates, qui ne voulait pas voir Washington en cette affaire, opposa un refus entêté; Lafayette tint bon; il menaça même de retourner en France, lui et tous les officiers étrangers qui l'accompagnaient ${ }^{37}$. Lafayette avait l'appui du président du Congrès, Henry Laurens.

32. Lafayette.

33. Conway.

34. Dès 1776, la politique de Louis XVI fut de laisser le Canada à l'Angleterre afin d'opposer davantage Britanniques et Américains; à partir du traité d'alliance de 1778 , c'est sur ce Canada britannique que la France compta surtout pour retenir les Américains dans son alliance.

35. LMCC III 50-51.

36. MacDougall était Américain; de Kalb était un baron allemand déjà venu en Amérique en 1768 faire de l'espionnage au service de Choiseul; il y revint en 1777 se mettre au service du Congrès: on ne le considérait pas comme un Français d'adoption, ainsi que l'était Conway.

37. Henry Laurens à John Rutledge, le 30 janvier 1778; LMCC III 64. 
Ce dernier, bien qu'opposé au projet d'invasion, mettait toute sa confiance en Lafayette: "Il n'y a personne dans notre armée ", écrivait-il, " qui ait, dans cette invasion, autant de chances de réussir que ce jeune noble. Il est habile dans l'art dè la guerre, il est sensé et brave et il aura une grande influence au Canada auprès de la noblesse, de l'Église et de la bourgeoisie. " ${ }^{3}$. Aidé de Laurens, Lafayette finit par l'emporter: " Espérant enivrer et conduire un commandant si jeune, le Bureau de la Guerre, sans consulter son commandant en chef, lui manda d'aller attendre ses instructions à Albany. Mais, après avoir persuadé le Comité du Congrès au camp, M. de Lafayette courut à Yorktown et y déclara: qu'il lui fallait des ordres détaillés, un état des moyens, la sécurité de ne pas tromper les Canadiens, une augmentation de généraux, des grades pour plusieurs Français... enfin la condition première de n'être pas, comme Gates, indépendant du général Washington. " ${ }^{3} 9$ Lafayette alla plus loin: lors d'un banquet présidé par Gates lui-même, on but à la santé du futur conquérant du Canada; quand vint le tour de Lafayette de proposer la santé, il fit remarquer aux convives qu'on avait oublié la plus importante, celle du général en chef Washington, et il " leur fit porter, en rougissant, la santé de leur général. " ${ }^{40}$ C'était un véritable défi au Bureau de lq Guerre.

Lafayette obtint donc de dépendre de Washington et celui-ci désigna un major-général de l'armée américaine pour accompagner Lafayette $^{41}$. Le jeune marquis obtint des commissions d'officiers pour les nobles de sa suite qui se proposaient de se mettre à la tête des volontaires enrôlés au Canada ${ }^{43}$, trente mille livres françaises qu'il

38. Id., op. cit., p. 65.

39. LAFAyetre, Mémoires (éd. Georges-Washington Lafayette, 1837), t. I, p. 39. Dans ses Mémoires, Lafayette parle toujours de lui-même à la troisième personne.

40. Lafayette, loc. cit.- Edmund C. Burnett, The Continental Congress, p. 292.

41. MacDougall fut d'abord désigné, puis, malade, fut remplacé par de Kalb (W Ashington, Writings, t. $X$, p. 428).

42. Pour la liste de ces officiers, voir SJCC I 58-59 et JCC X 107-108. On y remarque entre autres le nom de monsieur des Epiniers, neveu de Beaumarchais et promu major "en considération des services rendus par son oncle. "

43. Les prisonniers canadiens qui avaient fait partie de l'armée de Burgoyne tentaient tous les jours d'obtenir leur libération par l'entremise de Lafayette, qui en parle lui-même (The letters of Lafayette to Washington, éd. Gottschalk, 1944, p. 35). C'est probablement à cette occasion qu'il leur aurait répondu par ce mot qu'on cite de lui: "Puisque vous avez choisi d'être esclaves, restez-le ". 
pourrait dépenser comme il l'entendrait ${ }^{4}$; ses instructions qu'il voulut très précises, lui " promirent 2,500 hommes réunis a Albany, un gros corps de milices à Coos... tous les moyens de traverser sur la glace le lac Champlain, d'où, après avoir brûlé la flottille anglaise, il tomberait a Montréal et y agirait suivant les circonstances. " ${ }^{45}$ Pour compléter ces dispositions, on fit partir douze éclaireurs par la route de la Kennebeck et de la Chaudière afin d'annoncer qu'une invasion allait avoir lieu par cette route et qu'elle avait pour objectif Québec: ces éclaireurs devaient, pour donner plus de vraisemblance à la rumeur, s'informer des provisions que l'armée américaine pourrait obtenir sur son passage $^{46}$. On espérait ainsi amener les Britanniques du Canada à divertir une partie de leurs forces de Montréal pour défendre la route de la Chaudière.

Tout semblait donc prêt pour cette invasion et les Etats-Unis allaient, une deuxième fois, tenter de faire du Canada britannique le quatorzième Etat américain. L'occasion pouvait paraître belle. Un délégué du Congrès écrit que Gates a préparé l'invasion parce qu'il avait appris que les garnisons de Saint-Jean et de Chambly étaient très faibles, que les Canadiens étaient mal disposés envers les Britanniques, qu'il y avait à Montréal d'abondants magasins et qu'une irruption soudaine pourrait les faire tomber entre les mains des Américains ${ }^{4}$. Il y avait surtout nécessité pour les Etats-Unis de conquérir le Canada si l'on voulait un jour ou l'autre conclure avec l'Angleterre une paix qui serait fondée sur l'amitié ${ }^{48}$ : en effet, si à la paix le Canada restait à l'Angleterre, la paix ne serait en définitive qu'une paix armée. Cependant, l'invasion du Canada n'était pas vue partout d'un œil favorable. En certains milieux, on voulait que le Canada fât confédéré plutôt que conquis: conquis, il ajoutait à la puissance de la Nouvelle-Angleterre (c'est cette dernière en somme qui allait soutenir l'expédition) qui, un jour ou l'autre, serait tentée de subjuguer ensuite le reste du continent; confédéré, c'est-à-dire

44. SJCC I 57; JCC X 97.

45. Lafayete, Mémoires, t. I, p. 40. 213.

46. The Heath Papers (Mass. Hist. Soc., 7th series, vol. IV), part. II, p. 212-

47. Eliphalet Dyer à William Williams, le 17 février 1778: LMCC III 88-89.

48. Benjamin Franklin à James Hutton, le 1er février 1778: RDCor II 484. 
ajouté à la république américaine d'une façon volontaire et suivant certaines conditions, le Canada pourrait servir a tenir en échec la Nouvelle-Angleterre ${ }^{4}$. Quant au président du Congrès, nous avons déjà vu quelle était son opinion sur le plan de Gates.

Parmi les chefs militaires, plusieurs estimaient que l'invasion était vouée à un échec certain. Washington, laissé de côté lors de la présentation du projet au Congrès, avait dû être consulté à la dernière minute par le Bureau de la Guerre, à la suite des exigences de Lafayette; il répondit: "Dans le cas présent, comme je ne connais ni l'étendue des objectifs que vous avez en vue ni les moyens que vous emploierez pour les atteindre, il n'est pas en mon pouvoir de prononcer quelque jugement que ce soit. Je ne puis que souhaiter sincèrement que le succès l'accompagne, autant pour l'effet qu'il peut produire sur le progrès du bien public que pour l'honneur personnel du marquis de Lafayette que j'estime et considère d'une façon tout à fait particulière. " ${ }^{50}$ Consulté tardivement, alors que tout était décidé et supposé prêt, il ne pouvait guère répondre autre chose, se fiant à la prudence de Lafayette, mais dans une lettre à un ami, Washington condamna cette invasion qu'il qualifiait de " child of folly " ${ }^{51}$.

Schuyler avait sans doute ses raisons personnelles de s'opposer au projet de Gates, puisque Gates était son ennemi et que cet ennemi l'avait complètement ignoré en préparant l'invasion du Canada; il n'en reste pas moins que Schuyler, en écrivant à Conway " que dans les circonstances actuelles on ne pouvait pas commencer une entre-

49. Arthur Lee à Samuel Adams, le 28 février 1778: R.H. Lev, Life of Arthur Lee with his political and literary correspondence (Boston, 1829), t. II, p. 137.

Il faut se rappeler les divisions profondes qui séparaient les divers États de la nouvelle république; tous ces Etats n'étaient en réalité qu'autant de pays différents confédérés entre eux d'une façon fort précaire: leurs rivalités mirent souvent en péril l'existence de la république au cours même de la guerre de Révolution. On se souvenait d'ailleurs que de 1643 à 1684, grâce au Massachussetts, la NouvelleAngleterre était devenue une confédération agressive. Les Etats du Sud se défiaient de l'esprit de conquête des Etats du Nord et si l'invasion de 1775 fut si mal préparée et si peu soutenue par le Congrès, il faut en chercher la cause dans l'opposition présentée chaque jour par les délégués des États du Sud: ceux-ci avaient déjà assez de difficultés à tenir en équilibre la balance des pouvoirs, il ne fallait pas que le Canada, dont les intérêts étaient ceux de la Nouvelle-Angleterre, vînt faire pencher davantage le plateau du Nord.

50. Au Bureau de la Guerre, le 27 janvier 1778: Writings, t. X, p. 356.

51. A Thomas Nelson Jr., le 8 février 1778; ibid., p. 433. 
prise contre le Canada $"{ }^{52}$, parlait en connaissance de cause: général de l'armée expéditionnaire de.1775 et, qui plus est, général malheureux, il savait tout ce qu'une invasion du Canada pouvait présenter de difficultés, difficultés de commandement, difficultés de ravitaillement, difficultés financières et autres.

Arnold avait plus de raisons encore que Schuyler de se plaindre d'avoir été laissé de côté et plus encore que Schuyler il avait le droit d'être consulté sur ce projet d'invasion: de tous les officiers qui avaient survécu à l'invasion de 1775-76, c'est lui qui avait acquis la plus grande expérience dans ce domaine; sa marche héroïque de Newport à Québec et la ténacité extraordinaire qu'il avait manifestée dans les situations les plus critiques, donnaient le plus grand poids au jugement qu'il pouvait prononcer sur le plan de Gates. Or Arnold condamnait ce plan. C'est dans une lettre à Gouverneur Morris qu'il exposa le plus longuement ses objections ${ }^{53}$ : " J'ai été grandement surpris, il y a quelques jours, d'apprendre qu'une expédition était sur pied contre le Canada à cette époque tardive... Les personnes qui doivent commander les troupes de cette expédition, à ce qu'on me dit, sont le marquis de Lafayette et le général Conway: le premier, je n'ai pas l'honneur de le connaître; l'autre, je crois, est un officier de mérite et d'expérience, mais comme tous deux sont totalement étrangers au pays, ils ne peuvent avoir qu'une faible idée des difficultés qu'ils ont à rencontrer dans une campagne d'hiver. D'après les faibles connaissances que j'ai des nombreuses difficultés qui accompagnent inévitablement l'expédition, j'oserai présager qu'elle va avorter; en tout cas, les chances contre son succès sont de vingt contre une. ) Et il énumère avec facilité les motifs de ce pronostic défavorable: l'armée manque de provisions, de bagages, de raquettes, de moyens de transport, de tout; les habitants du Canada n'ont jamais de viande salée et ils n'ont plus de viande fraîche, parce qu'ils se sont vus enlever leurs animaux par les troupes britanniques; surprendre Saint-Jean est une supposition absurde: s'il y a cinq cents hommes pour le defendre, dix mille hommes ne pourront s'en emparer avec seulement l'artillerie légère; or il est impossible de transporter de l'artillerie lourde en hiver; si l'expédition

52. Lafayette, Mémoires, t. I, p. 157.

53. Le 24 février 1778. Charlemagne Tower, The Marquis de La Fayette in the American Revolution, t. II, appendice B, p. 479-481. 
échoue, elle devra compter sur ses propres vivres et sur ses propres forces pour retraiter: et comment opérer une retraite à l'époque du dégel ? Arnold ajoute: " Si le dessein du Congrès n'est que de prendre possession de Montréal, je doute fort qu'il trouve cet objet digne de la dépense "); il ne voit d'ailleurs aucune nécessité d'entreprendre l'invasion cette année, puisque les Britanniques du Canada ne peuvent plus songer à envahir les Etats-Unis par le nord et que, une fois les Britanniques chassés du territoire américain, le Canada tombera de lui_même " probablement sans le risque ni les frais d'une expédition "). Enfin, Arnold ne manque pas de voir au fond de tout cela des intrigues personnelles: " Je suis entièrement persuadé que le Congrès a été précipité dans cette mesure par des hommes entreprenants ou ignorants qui n'ont pas sur cette affaire les connaissances nécessaires. ")

Arnold, en énumérant les difficultés du projet, savait ce dont il parlait: il se souvenait que le fort Saint-Jean avait tenu près d'un. mois et demi contre l'artillerie lourde des Américains, que sa résistance avait eu pour résultat de retarder à l'hiver les opérations contre Québec; il savait par expérience que le dégel du printemps était une époque où toute communication devenait impossible entre les EtatsUnis et le Canada; il se rappelait sans doute aussi les violences qu'il avait dû exercer lui-même sur les habitants pour en obtenir des vivres, ainsi que l'effet inutile qu'avait produit la prise de Montréal pour déterminer la possession du Canada; il était convaincu, et bien d'autres officiers et bien des hommes d'Etat l'étaient aussi, que l'Angleterre n'oserait jamais tenter de nouveau la marche de Burgoyne; enfin, lorsque Arnold dit que deux étrangers venus d'Europe ne peuvent pas s'imaginer les embarras d'une campagne d'hiver, c'est le simple bon sens qui parle par sa bouche.

Quant à Lafayette, il accepta l'entreprise avec un mélange d'enthousiasme et d'inquiétude. Il écrivait à sa femme, le 3 février: " Je ne vous ferai pas de longs détails sur la marque de confiance dont l'Amérique m'honore. Il vous suffira de savoir que le Canada est opprimé par les Anglais; tout cet immense pays est en possession des ennemis, ils y ont une flotte, des troupes et des forts. Moi, je vais m'y rendre avec le titre de général de l'armée du nord et à la tête de 3,000 hommes pour voir si l'on peut faire quelque mal aux Anglais dans ces contrées. L'idée de rendre toute la Nouvelle-France libre et de la délivrer d'un joug pesant, est trop brillante pour s'y arrêter. 
Alors mon armée augmenterait immensément et serait augmentée par des Français. " ${ }^{54}$ Mais cet enthousiasme ne se prolonge guère: " J'entreprends un terrible ouvrage, surtout ayant peu de moyens. Quant à ceux de mon propre mérite, ils sont bien nuls pour une telle place, et ce n'est pas à vingt ans qu'on est fait pour être à la tête d'une armée... J'ai une immensité d'affaires, et il y a une infinité de choses politiques et militaires à arranger; il y a tant de choses à réparer, tant de nouveaux obstacles à lever, qu'en vérité il me faudrait quarante ans d'expérience et des talents supérieurs pour ne pas en sortir avec désagrément. " ${ }^{55}$

Rien de bien brillant ne s'annonçait donc et Lafayette, l'esprit rempli de sombres pensées, se mit en route pour Albany où il devait trouver ses troupes. Au cours du trajet, il écrit à Washington, le 9 février: " J'avance très lentement, tantôt percé par la pluie, tantôt couvert de neige, et ne m'entretenant pas de bien riantes idées sur l'incursion projetée en Canada. S'il y avait des succès, ce serait pour moi une surprise très agréable, par la raison que je n'en attends point de fort brillants. Le lac Champlain est trop froid pour produire le moindre brin de laurier, et, si je ne suis pas noyé ou ne meurs pas de faim, je serai aussi fier que si j'avais gagné deux batailles. " ${ }^{56}$ Duer, délégué du Congrès, et Thomas Conway lui avaient donné rendez-vous dans une taverne, mais ne s'y étaient point trouvés et Lafayette écrit, essayant encore de badiner: "Peut-être conquerront-ils le Canada avant mon arrivée et je m'attends à les trouver à l'hôtel du gouverneur a Québec. " ${ }^{57}$ Des déceptions plus amères l'attendaient a Albany: "Je défie Votre Excellence ", écrit-il à Washington le 19 février, " de concevoir une idée de ce que j'ai vu, depuis que j'ai quitté le lieu où j'étais tranquille auprès d'un ami, pour me jeter au milieu de toutes les bévues de la sottise ou de la perfidie, Dieu sait laquelle. /) ${ }^{58}$ Il se heurte à Conway dont " le premier mot a été que l'expédition était impossible "); il y apprend que Schuyler, Arnold et Lincoln

54. Lafayette, Mémoires, t. I, p. 151s.

55. Ibid., p. 152.

56. Ibid., p. 154.

57. Loc. cit.

58. Ibid., p. 156: 
condamnent d'avance l'expédition; Hazen lui-même ${ }^{59}$ qui attendait depuis deux ans l'occasion de rentrer en son pays, avouait qu'on n'était pas assez fort " pour penser à l'expédition en ce moment "; les troupes sont dégoûtées, fait encore remarquer Lafayette, " effrayées au plus haut point d'entreprendre une incursion d'hiver dans un pays si froid... Il règne un esprit de mécontentement parmi les soldats et même les officiers; et cela tient à ce que depuis un temps énorme, ils n'ont point été payés ". On manquait donc de tout avant même que de partir. De plus, on n'avait rien préparé, explique-t-il à Washington, toujours dans la même lettre du 19 février: "J'ai été induit en erreur par le Bureau de la Guerre; on m'avait promis 3,000, et ce qui est plus positif, garanti par écrit 2,500 combattants, au calcul le plus faible. En ce moment, je n'en compterais pas en tout 1,200 propres au service, et la plupart sont comme nus, même pour une campagne d'été. Je devais trouver le général Stark avec un corps considérable... Eh bien, la première lettre que je reçois à Albany est du général Stark qui veut savoir quelle quantité, de quel lieu, pour quel temps, pour quel rendezvous je désire qu'il recrute du monde... Depuis deux jours, je m'adresse à tout le monde, je frappe à toutes les portes et je vois qu'il serait possible de faire quelque chose si l'expédition devait commencer dans cinq semaines. Mais vous savez que nous n'avons pas une heure à perdre, et même à présent, si tout était prêt, il serait presque trop tard. ") 60

Ainsi donc, alors qu'il fallait envahir tout de suite, par surprise et avant la fonte des neiges, rien n'était prêt: l'armée régulière manquait de tout le nécessaire et la milice n'était pas encore convoquée. Le Bureau de la Guerre n'avait pas exécuté ses promesses. Que restaitil à faire à ce général de vingt ans, " animé par l'attente de l'Amérique et bientôt celle de l'Europe ") ${ }^{61}$ et rêvant d'acquérir la gloire des armes? Lorsque le Congrès avait remis ses instructions à Lafayette, il lui avait recommandé de déterminer sa conduite sur les chances de succès et de n'exposer ses troupes à aucun hasard sérieux ${ }^{62}$; or, dans les

59. Moses Hazen, de Saint-Jean, après avoir pris parti pour les Américains lors de l'invasion de 1775 , avait dú les suivre dans leur retraite. Il avait été fait colonel de l'armée américaine.

60. LAfaymtte, Memoires, $t, I, p .156-158$.

61. Ibid., p. 42.

62. Instruction du 24 février 1778: SJCC I 60; JCC X 193. 
circonstances présentes, Lafayette ne pouvait absolument pas aller de l'avant, a moins de risquer l'extermination de ses propres troupes, persuadé d'ailleurs que " le lieutenant-général Carleton lui préparait un autre Saratoga $1{ }^{63}$. Mais il lui en coutait énormément de renoncer à l'entreprise à cause de la honte qui pourrait s'ensuivre pour lui: " Je vous ai dernièrement, " écrit-il à Washington, " rendu compte de mon affligeante et ridicule situation, qui réellement n'a pas de nom. Je suis envoyé avec grand bruit à la tête d'une armée pour faire de grandes choses. Le continent, et bientôt la France, l'Europe, et qui pis est, l'armée anglaise sont dans l'attente. Comme ils seront trompés, combien on se rira de nous... Dès que je veux regarder quelque part, je suis sûr qu'on étend un nuage devant mes yeux. Cependant, il y a des points sur lesquels on ne peut me tromper: le manque d'argent, le mécontentement des soldats, la répugnance de tous pour l'expédition, a l'exception des Canadiens qui s'imaginent rentrer chez eux; tout cela est aussi clair que possible. Je crains la risée. " ${ }^{64}$ Toutefois, chez ce général de vingt ans, la prudence fut plus forte encore que l'enthousiasme et "en gémissant ${ }^{65}$, il renonça à l'expédition. C'était à la fin de février.

De son côté, le Congrès, réuni en session à Georgetown (Maryland) ${ }^{66}$, avait reçu un rapport du Bureau de la Guerre et en était venu à la conclusion que le projet contre le Canada était " non seulement hasardeux à un haut degré mais extrêmement imprudent ": il adopta une résolution qui donnait ordre à Lafayette de suspendre l'expédition $^{67}$. C'est le 2 mars qu'on adopta cette mesure: à ce moment, l'expédition de Lafayette devait être en marche; il était peut-être trop tard pour la contremander. On dépêcha en toute hâte des courriers à Lafayette dans l'espoir qu'il serait encore temps de le rejoindre et de sauver d'un désastre certain l'armée américaine qu'on lui avait confiée. Mais lorsque les courriers parvinrent à Albany, il y avait déjà plusieurs jours que Lafayette avait renoncé à son projet: cet adolescent,

63. Lafayette, loc. cit.

64. Le 23 février 1778: LafayetTF, op. cit., t. I, p. 160.

65. Lafayette, ibid., p. 42.

66. Le Congrès avait dú quitter Philadelphie'lors de l'arrivée des Britanniques en septembre 1777; il ne devait y rentrer qu'en juillet 1778.

67. SJCC I 61; JCC X 216-217. 
que toute gloire militaire aurait dû éblouir, avait devancé le Congrès par sa prudence.

Washington lui écrivit pour le féliciter et le réconforter: "Vous semblez craindre que le monde ne laisse tomber sur vous un blâme proportionné à son attente déçue, par suite de l'abandon de l'expédition du Canada. Mais d'abord je ne vois rien de fâcheux pour vous à ce qu'il soit connu en Europe que vous avez reçu du Congrès une éclatante preuve d'estime et de confiance dans l'important commandement d'un corps détaché; et je suis persuadé que tout le monde approuvera la prudence qui vous a fait renoncer à une entreprise dont la poursuite vous eât engagé dans une lutte vaine contre des impossibilités physiques. " " ${ }^{68}$ Gates lui-même félicita Lafayette ${ }^{69}$; en renonçant à exécuter un projet que le Bureau de la Guerre n'avait même pas pris la peine de préparer, Lafayette, en somme, avait sauvé de la honte Gates et les officiers de son clan;mais il ne manqua pas d'attribuer à qui de droit, c'est-à-dire à Gates, la machination de toute l'affaire: "A présent, confie-t-il à Washington, nous connaissons le but que se proposait l'honorable Bureau, et pour quel résultat trois ou quatre hommes auraient entraîné le pays dans de grandes dépenses, risqué la réputation de nos armées et la vie de plusieurs centaines d'hommes si le général, votre ami, qu'on voulait tromper, eût été aussi imprudent et aussi insensé qu'on semblait l'espérer. " " 70

Malgré le secret dont on avait essayé d'envelopper les préparatifs de l'invasion, la nouvelle s'en était quand même répandue en certains milieux et avait donné naissance à des rumeurs qui répondaient plus aux désirs des intéressés qu'à la réalité. Allen Jones, délégué de la Caroline septentrionale au Congrès général, écrivait le 31 janvier

68. Washington à Lafayette, le 10 mars 1778: Washington, Writings, t. XI, p. 59; lettre traduite par Georges-Washington Lafayette dans les Mémoires de Lafayette, t. I, p. 162.

69. Lafayette, Mémoires, t. I, p. 42 , n.

70. LAFAYETTE, op. cit., t. I, p. 166. La faillite de cette intrigue fut pour Gates le début de la chute; il allait peu à peu perdre de son crédit en attendant de connaître, lors de la bataille de Camden, en 1780, la plus profonde humiliation (Gates couronna sa défaite en désertant honteusement son armée qui fuyait). Jugé devant un conseil de guerre et remplacé par le général Greene, il disparut de la scène militaire de la façon la plus silencieuse. 
1778: " Un monsieur de Virginie m'a dit hier avoir vu une personne, venue du nord, qui affirme qu'on peut être certain que les Canadiens ont consenti à se confédérer avec les treize Etats-Unis et que leurs représentants sont rendus au Congrès. Je souhaite de tout cœur que tout cela puisse être vrai. " ${ }^{71}$ Richard Henry Lee écrit de son côté, le 6 février 1778: " Je suis sans nouvelle aucune à l'exception de celle qui se répand au sujet des Canadiens et qui veut qu'ils se soient révoltés contre les Anglais, qu'ils aient pris Saint-Jean, Québec, etc. Cette nouvelle vient d'une source si directe et par des voies si sûres que je commence à y ajouter foi. " ${ }^{72}$ Dans une lettre de Versailles, écrite le 6 juin de la même année par un courtisan occupé à noter tous les commérages sur la grossesse de la reine ou de Madame, nous relevons le passage suivant: " Il y a des gens ici qui prétendent qu'un corps d'armée américaine, dans lequel $M$. de la Fayette sert avec son régiment, est entré dans le Canada, s'est rendu maître de Québec, dont les bourgeois s'étaient soulevés à temps. Mais cette nouvelle a grand besoin de confirmation. " ${ }^{73}$ La confirmation n'allait jamais venir...

S'il était permis à celui qui écrit l'histoire de se représenter, par pure complaisance, le passé autrement qu'il ne fut, nous pourrions nous poser la question suivante: si l'invasion de Lafayette, bien préparée, avait été menée avec le même succès que celle de Montgomery à l'automne de 1775, quelle réception les Canadiens lui auraient-ils faite? On sait qu'en 1775 les classes dirigeantes de la nation (le clergé, la noblesse, la bourgeoisie) s'étaient opposées à l'invasion américaine en refusant de collaborer ${ }^{74}$ : l'envahisseur américain représentait alors l'ennemi traditionnel de la Nouvelle-France, celui qui avait le plus contribué à la conquête de 1759; cet envahisseur s'était révolté contre

\section{LMCC III 65n.}

72. The letters of Richard Henry Lee (éd. Ballagh), t. I, p. 386.

73. Correspondance secrète inédite sur Louis XVI, Marie-Antoinette, la Cour et la Ville de 1777 d 1792 (éd. Lescure), t. I, p. 174.

74. Le clergé, la noblesse et la bourgeoisie, comme groupes, se sont montrés opposés à l'invasion américaine: les autorités ecclésiastiques ont agi sévèrement contre les collaborateurs, la noblesse s'est montrée très active dans l'organisation de la défense militaire, la bourgeoisie s'est montrée neutre. Il y eut évidemment des exceptions, très rares dans le clergé et dans la noblesse, beaucoup plus nombreuses chez les marchands (presque tous des Anglais et dont un bon nombre venait des colonies américaines). 
le Parlement anglais, ce même Parlement qui venait de favoriser singulièrement les Canadiens, mais il ne parlait pas encore de rompre son allégeance britannique: comment les Canadiens auraient-ils pu être tentés d'unir leur sort à des colonies révoltées s'il s'agissait toujours de rester soumis à la Couronne? En cette année 1778, les positions n'étaient plus les mêmes: si, à vrai dire, le prestige américain au Canada était fort en baisse depuis les derniers mois d'occupation (mai et juin 1776), les Canadiens pouvaient avoir de fortes tentations de changer de sentiments à l'égard des Américains: ceux-ci s'étaient déclarés indépendants de la Couronne britannique; l'espoir pour les Canadiens de se libérer de l'Angleterre et de constituer un peuple libre, même si pour cela il fallait entrer dans une confédération avec les colonies voisines, pouvait certainement présenter à la population un objet fort alléchant. De plus, celui qui s'avançait à la tête de cette invasion, n'était ni un Américain ni un protestant, mais un Français, un noble de la France catholique, qu'accompagnait toute une suite d'officiers français; l'arrivée de Lafayette, e'était le retour des fleurs de lis qui ravivait dans les cœurs le souvenir.des brillants faits d'armes accomplis par Montcalm et Lévis dix-huit ans plus tôt. Libres de choisir entre Cramahé et Lafayette, entre Georges III qui les avaient conquis et un Congrès américain qui leur envoyait des nobles de France, quelle aurait été la conduite des Canadiens? Mais tout cela est de l'histoire qui ne s'est point faite et qu'il faut renvoyer au royaume des rêveries.

L'invasion projetée par le Bureau de la Guerre et si mal préparée n'eut pas lieu. C'était la première tentative américaine depuis la défaite de 1776 et elle allait être suivie de plusieurs autres. Lafayette surtout allait tenter plusieurs fois encore de réussir son projet favori, reconquérir le Canada. Le projet du printemps de 1778 pouvait paraitre simple: le Congrès, dans sa politique extérieure, n'avait encore à tenir compte d'aucune puissance alliée ${ }^{75}$, mais c'est là le dernier projet qu'il put ainsi concevoir indépendamment de qui que ce fât. Le traité d'alliance conclu avec la France allait modifier considérablement la politique internationale: le Congrès ne pourrait plus songer au Canada sans tenir compte de la politique de Louis XVI; or la France allait manœuvrer pour que les Britanniques restent maîtres

75. Le traité d'alliance, signé le 6 février avec la France, ne fut annoncé publiquement que le 20 mars et la nouvelle n'en parvint au Congrès que le 2 mai. 
du Canada. Le projet d'invasion du Canada, en février 1778, était pour le Congrès la dernière chance d'en finir de lui-même et seul, avec le Canada britannique: le Congrès ne fut pas plus heureux qu'en 1776 .

\author{
Marcel TrudeL, \\ charge de cours a l'Institut d'histoire \\ et de géographie de l'Université Laval
}

Tous droits réservés 